\title{
Characterization of Bowel Lesions with Multi-detector CT Scan
}

\author{
Kalpesh K. Patel ${ }^{1}$, Mayur V. Khandhedia ${ }^{2}$, Vishalkumar H. Bhardava ${ }^{3}$ \\ ${ }^{1}$ Assistant Professor, ${ }^{2}$ Associate Professor, ${ }^{3}$ Assistant Professor, Department of Radiology, Dhiraj Hospital, SBKS Medical \\ Institute and Research Centre, Sumandeep Vidhyapeeth University, Pipariya, Vadodara, India
}

Corresponding author: Mayur V. Khandhedia, 2, Kanha Bunglow, Opp. Vrundavan bunglow, Kubereshwar Marg, WaghodiaDabhoi Ring Road, Vadodara-390019, India

DOI: $10.21276 / \mathrm{ijcmsr}$.2018.3.3.30

How to cite this article: Kalpesh K. Patel, Mayur V. Khandhedia, Vishalkumar H. Bhardava. Characterization of bowel lesions with multi-detector CT scan. International Journal of Contemporary Medicine Surgery and Radiology. 2018;3(3):C136-C142.

\section{A B S T R A C T}

Introduction: This study aims to characterize various bowel pathologies, as well as usefulness of Multidetector CT scan in differentiating benign versus malignant lesions and to evaluate CT findings with histological data.

Material and methods: This prospective study was done in radiology department of Dhiraj Hospital, SBKSMIRC, Sumandeep Vidhyapeeth University, Vadodara, India during period of April, 2015 to September, 2017.94 patients with clinical presentation of bowel, mesentery and related pathologies were selected and underwent CT scan. Diagnosis was confirmed by following up patient with USG or CT, Other radiological and non-radiological investigation and/or surgery and histopathology.

Results: The mean age of patients was 50 years. Pathology is more common in male (61) as compared to females (33). Among the 94 patients included in the study, 2 had congenital lesions, 26 had infective and inflammatory lesions, 5 had ischemic bowel conditions, 54 had neoplastic lesions of bowel and 7 had miscellaneous bowel conditions. Colorectal malignancy is most common (82.36\%) malignant lesion with rectosigmoid region (52.38\%) as most common area. Differentiation of benign from malignant lesions can be done by certain characteristics of the lesions at CT scan, like degree of wall thickening, symmetry of wall thickening, length of wall thickening and postcontrast enhacement pattern.

Conclusion: No single characteristic is highly specific for differentiation between benign and malignant lesions. Careful analysis of characteristics of lesions on CT scan helps to narrow down the differential diagnosis. Thus, multidetector CT scan is "The imaging modality of choice" for characterization of bowel lesions.

Key words: Degree of Wall Thickening, Symmetry of Wall Thickening, Length of Wall Thickening, Postcontrast Enhancement Pattern

\section{INTRODUCTION}

We have studied bowel, mesentery and associated structures in with Multidetector CT scan machine.With the advent of Multidetector CT scan, it became possible to achieve very good quality images of bowel wall to detect subtle lesions of bowel wall as well as mesentery. The high speed of acquisition of volumetric data allows us to perform excellent quality CT angiography of mesenteric and abdominal vessels. Multidetector CT technology makes possible the acquisition of isotropic data and affords the capability of performing high resolution multiplanar reconstructions. ${ }^{3}$

This study aims to characterize various bowels, mesentery and related pathologies from duodenum to rectum with Multidetector CT scan, to correctly distinguish between benign from malignant lesions, to classify bowel pathologies according to etiology. This study also aims to evaluate role of Multidetector CT angiography of abdominal vessels in ischemic bowel conditions and to detect regional lymph node and abdominal distant metastases in cases of malignant bowel lesions. Optimal evaluation of an abnormal small bowel loop is facilitated when the small bowel is well distended, IV contrast material administered and thin section CT is used. ${ }^{6}$

\section{MATERIAL AND METHODS}

This prospective study was done in radiology department of Dhiraj Hospital, SBKS medical institute and research centre, Sumandeep Vidhyapeeth University, Pipariya, Vadodara, India during period of April, 2015 to September, 2017. The study was started after approval from Ethical Committee. Written informed consent from all participants was obtained. 94 patients with clinical presentation of bowel, mesentery and related pathologies arising from duodenum to rectum were selected for this study and underwent Multidetector CT scan.Diagnosis was confirmed by following up patient with USG or CT, Other radiological and non-radiological investigation and/or surgery and histopathology.

\section{Scanning and methods used}

CT scan of abdomen and pelvis was performed on Siemens 16 slice Multidetector CT scanner machine.Scanning was performed with acquisition of volumetric data from diaphragm to anal canal with pitch kept usually 1 with minimal slice thickness $0.625 \mathrm{~mm}$. Reconstruction algorithm used to obtain $1 \mathrm{~mm}$ voxels in axial, coronal and sagittal planes and also in multiple planes.Patients were asked to 
drink 800 to $1000 \mathrm{ml}$ of Mannitol or nonionic oral contrast before the scan. Mannitol was preferred as negative contrast agent. Each patient was administered 1 to $1.5 \mathrm{ml} / \mathrm{kg}$ body weight nonionic intravenous contrast (Iohexol 350 $\mathrm{mg} / \mathrm{ml}$ ) through power injector at rate of $2.5-3 \mathrm{ml} / \mathrm{sec}$. For CT angiography, 2 to $2.5 \mathrm{ml} / \mathrm{kg}$ body weight nonionic contrast was administered at rate of 3.5 to $4 \mathrm{ml} / \mathrm{sec}$. Images were acquired in noncontrast and multiple postcontrast phases as per requirement, e.g. triple phase or arterial and venous phases. Triple phase scanning was done after administration of intravenous contrast, in patients suspected of having liver metastasis on other investigation.

\section{CT scan analysis}

CT scan of patients with bowel, mesentery and related pathologies were analyzed on Siemens workstation.Bowel from duodenum to rectum was evaluated for bowel mass or wall thickening, degree of thickening, concentric or eccentric wall thickening, length of involved segment, attenuation, associated abnormalities, bowel dilatation and adjacent loculated fluid collections.

\section{Criteria}

Patients, whose clinical presentation suggested bowel pathology arising from the duodenum to the rectum where MDCT examination was done as investigational work up.

All patients who could be rescanned with USG/CT for follow up or followed for surgical / histopathological confirmation. Patients who could not be followed up to the final diagnosis are excluded.

\section{STATISTICAL ANALYSIS}

All the results were recorded in Microsoft excel sheet and analysed manually using statistical formulas for sensitivity, specificity, positive predictive value and accuracy.

\section{RESULTS}

The mean age \pm standard deviation of patients was $50.00 \pm$ 15.99 years (range: $14-85$ years). Pathology is more common in male $61(64.90 \%)$ as compared to females $33(35.10 \%)$.

Among the 94 patients included in the study, 2(2.13\%) had congenital lesions, 26(27.66\%) had infective and inflammatory lesions, 5(5.32\%) had ischemic bowel conditions, 54(57.44\%) had neoplastic lesions of bowel and $7(7.45 \%)$ had miscellaneous bowel conditions. (Chart 1)

Most commonly involved part of the bowel was colon

Common types of bowel pathologies $2 \%$

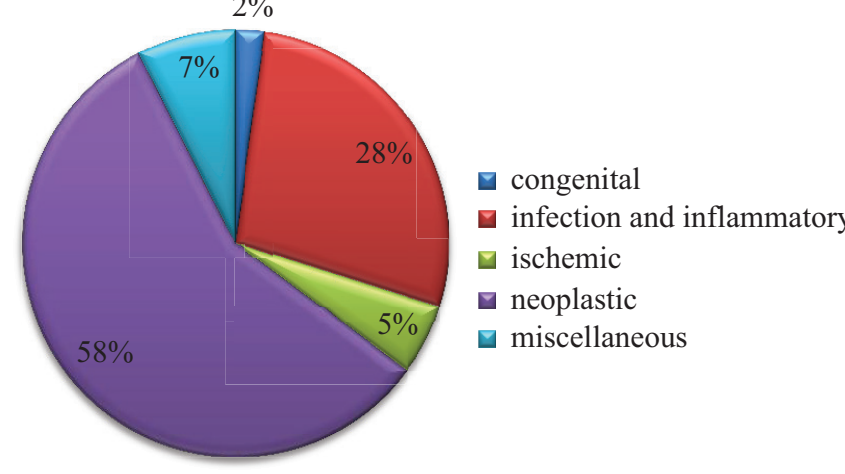

Chart-1: Types of bowel pathologies and rectum (52.13\%) followed by ileocaecal junction and caecum(18.08\%).Most common benign lesions are inflammatory colitis and granulomatous lesions. (39.53\%). Adenocarcinoma is most common malignant lesion (94.12\%).Two cases of intussusceptions were noted that were both in adult patients and associated with mass as their lead point. Lymphoma of ileum was detected in only one case. Ileal carcinoids were detected in two patients. Commonest benign lesion involving ileocaecal region is tuberculosis.

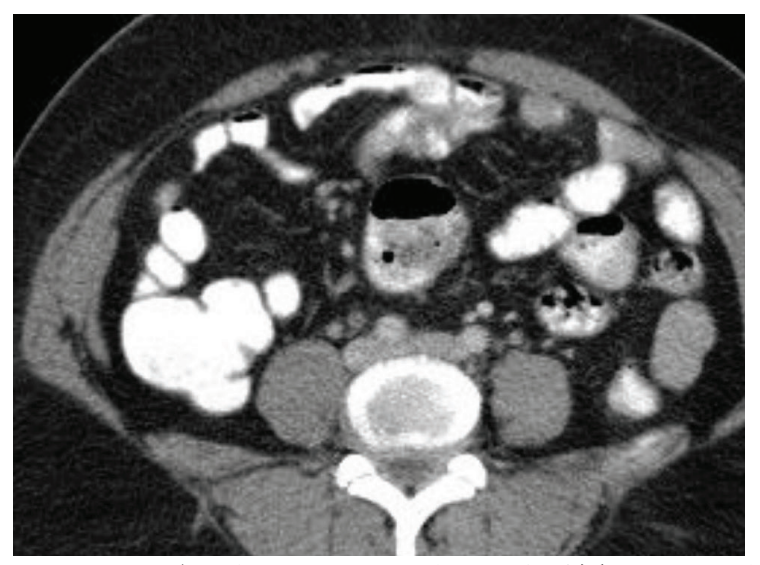

Figure-1: Axial contrast enhanced CT image showing normal wall thickness of large and small bowel (oral and perrectal contrast administered).

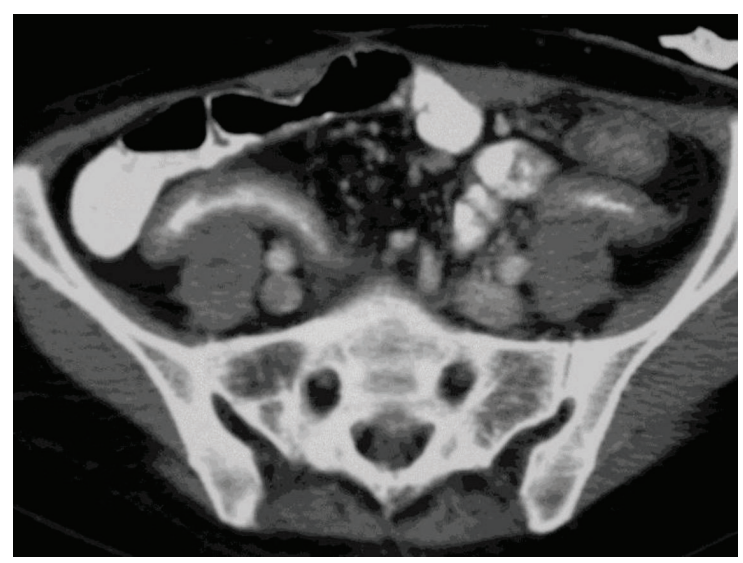

Figure-2: Axial contrast enhanced CT image showing mild symmetric wall thickening of ileum - Intestinal tuberculosis.

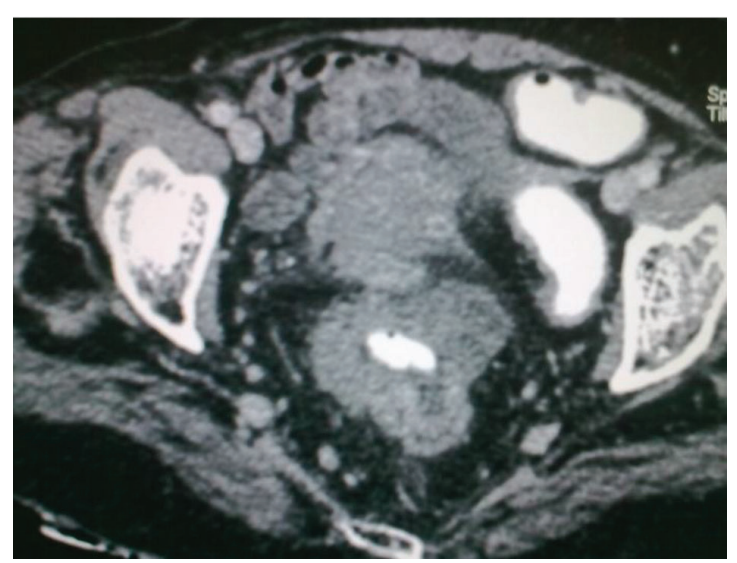

Figure-3: Axial contrast enhanced CT image showing marked asymmetrical wall thickening of rectum with heterogeneous postcontrast enhancement - adenocarcinoma 


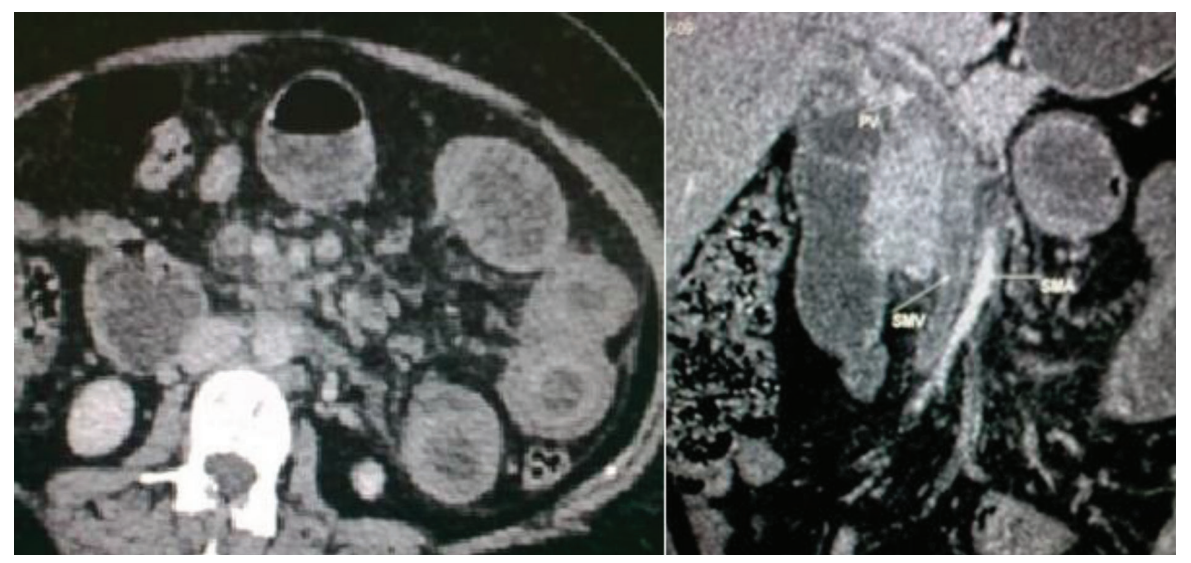

Figure-4 (a, b): (a)Axial contrast enhancend CT image showing symmetrical wall thickening of ileal loops with "target sign" (b) Coronal venous phase CT images showing thrombosis in superior mesenteric vein and portal vein.

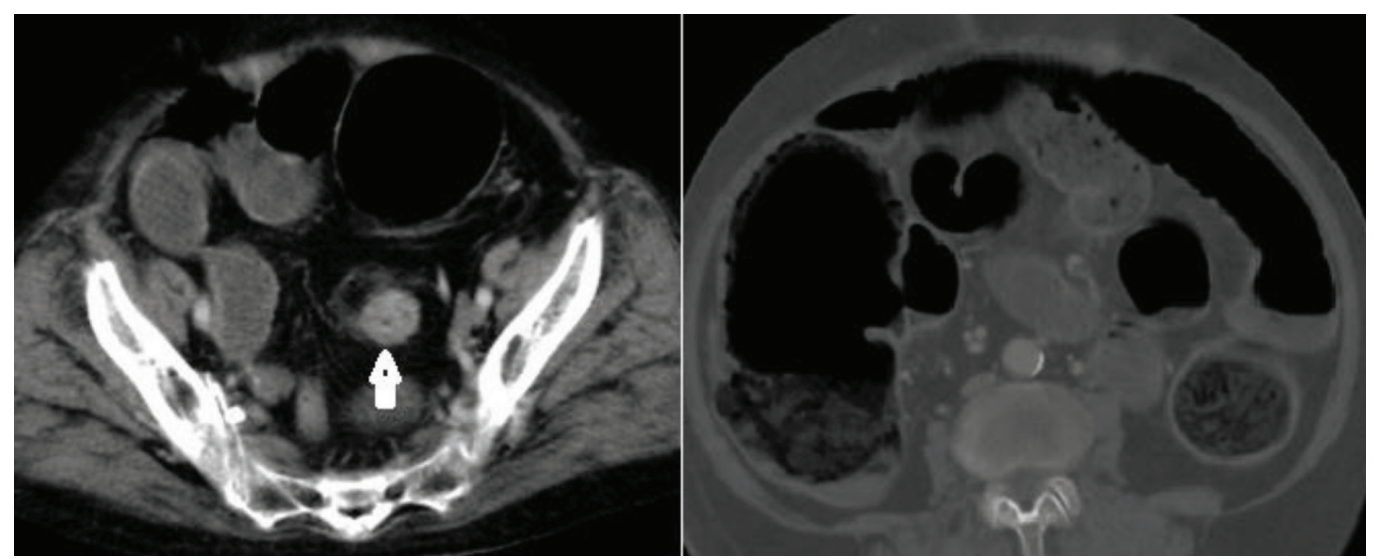

Figure-5 (a, b): Axial contrast enhanced CT images (a) showing symmetrical circumferential wall thickening of sigmoid colon (arrow) causing severe stricture and (b) showing obstruction of proximal large bowel and small bowel.
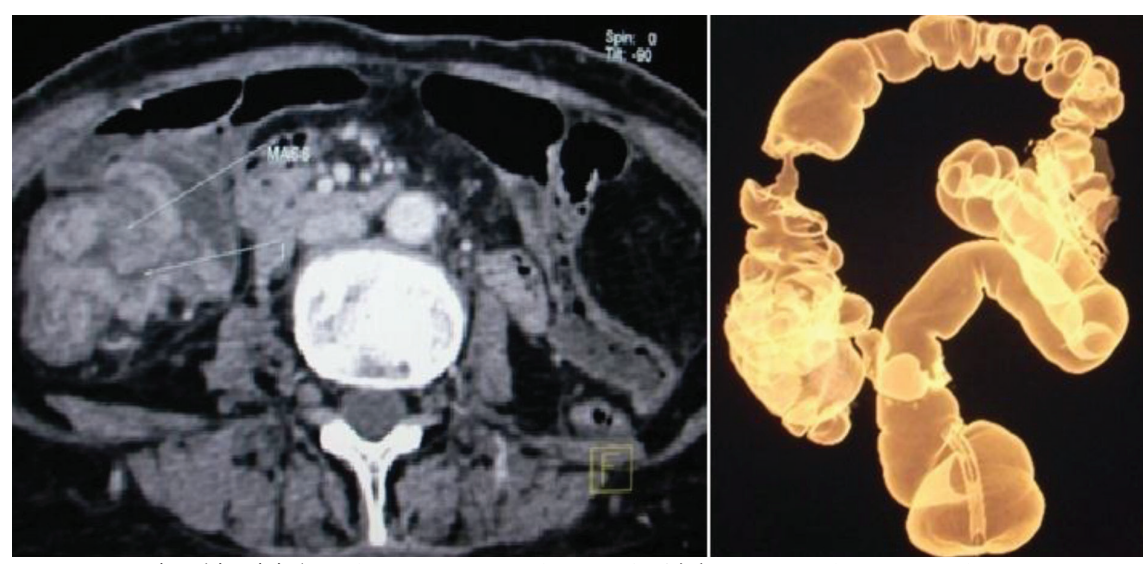

Figure 6 (a, b): (a)Axial contrast enhanced CT image showing short segment asymmetrical wall thickening involving ascending colon. (b) 3D virtual volume rendered image of CT colonography showing short segment irregular lesion with shouldering at either ends and causing stricture.

Colorectal malignancy is most common (82.36\%) malignant lesion with rectosigmoid region $(52.38 \%)$ as most common area followed by caecum and ascending colon(26.19\%). One patient had lymphoma of small bowel involving ileum showing significant segmental wall thickening with lymphadenopathy and splenomegaly. Bowel wall thickness was statistically significantly more in the malignant lesions compared to inflammatory, infective or ischemic lesions. Out of 5 patients with ischemic colitis, CT angiography detected superior mesenteric artery thrombosis in four patients and portal vein thrombosis and superior mesenteric vein thrombosis in one patient.

Out of 51 cases of adenocarcinoma of bowel, 41 (80.39\%) showed marked $(>1.5 \mathrm{~cm})$ bowel wall thickening, $43(84.31 \%)$ cases showed asymmetrical bowel wall thickening. Out of 18 cases of infective/ inflammatory bowel lesions, 15 (83.33\%) cases showed mild $(<1.5 \mathrm{~cm})$ bowel wall thickening and 14 $(77.77 \%)$ cases showed symmetrical bowel wall thickening. (Table 1)

Out of 51 cases of adenocarcinoma of bowel, 42 (82.35\%) 


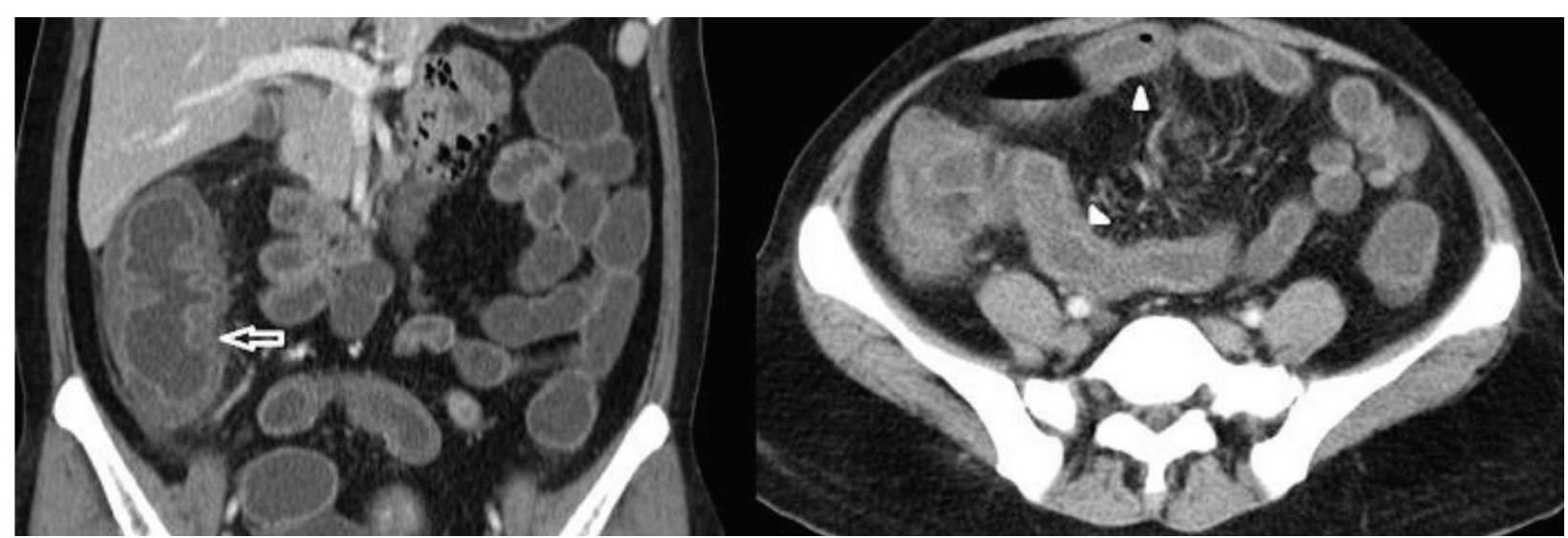

Figure-7 (a, b): Contrast enhanced coronal CT image showing long segment wall thickening of ascending colon and caecum (arrow). (b) Contrast enhanced axial CT scan image showing long segment ileal wall thickening in the same patient (arrowheads). Crohn' disease

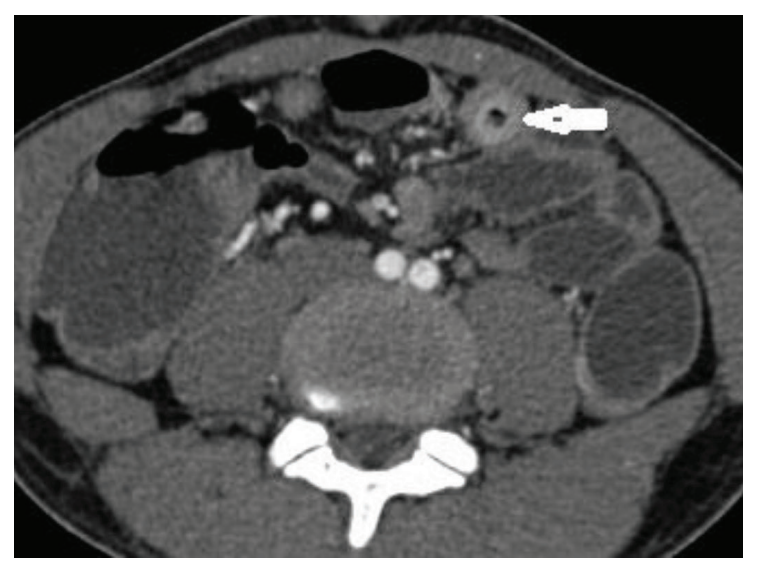

Figure-8: Axial contrast enhanced CT scan image showing "Halo sign" (arrow) - Crohn's disease.

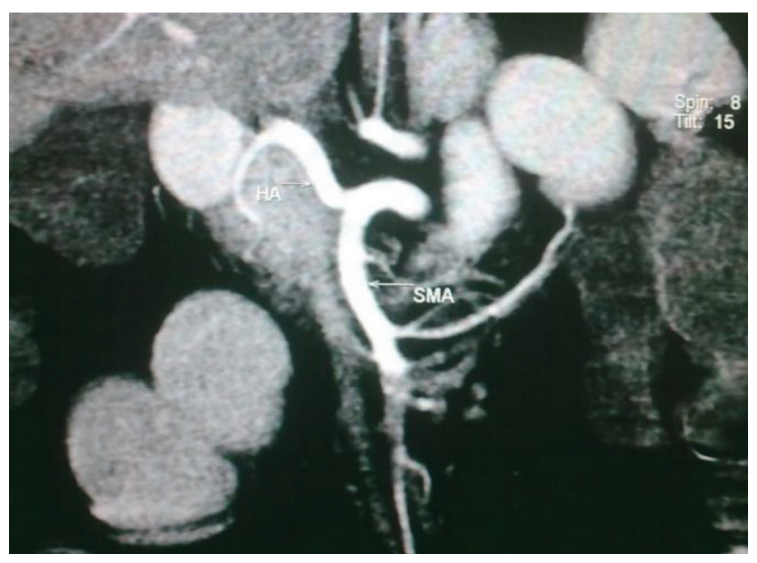

Figure-9: Coronal Maximum Intensity Projection (MIP) CT image in arterial phase showing thrombus in superior mesenteric artery.

\begin{tabular}{|c|c|c|c|c|c|c|c|c|c|}
\hline & & 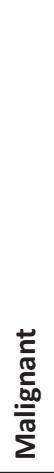 & 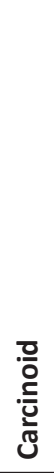 & 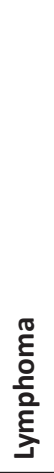 & 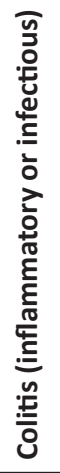 & 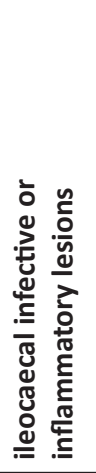 & 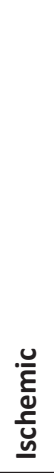 & 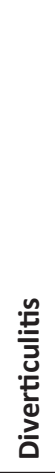 & $\begin{array}{l}\overline{\sqrt{0}} \\
\stackrel{0}{\circ}\end{array}$ \\
\hline \multirow[t]{2}{*}{ Degree of wall thickening } & Mild $(<1.5 \mathrm{~cm})$ & 10 & 2 & 0 & 5 & 10 & 4 & 1 & 32 \\
\hline & Marked $(>1.5 \mathrm{~cm})$ & 41 & 0 & 1 & 1 & 2 & 1 & 1 & 47 \\
\hline \multirow[t]{2}{*}{ Symmetry of wall thickening } & Symmetrical & 8 & 2 & 1 & 4 & 10 & 5 & 0 & 30 \\
\hline & Asymmetrical & 43 & 0 & 0 & 2 & 2 & 0 & 2 & 49 \\
\hline \multirow[t]{3}{*}{ Length of involved segmnent } & Focal & 42 & 2 & 0 & 1 & 3 & 0 & 2 & 50 \\
\hline & Segmental & 9 & 0 & 1 & 3 & 9 & 4 & 0 & 26 \\
\hline & Diffuse & 0 & 0 & 0 & 2 & 0 & 1 & 0 & 3 \\
\hline \multirow[t]{4}{*}{ Post-contrast enhance-ment pattern } & Homogeneous & 20 & 2 & 1 & 2 & 4 & 2 & 0 & 31 \\
\hline & Homogeneous & 31 & 0 & 0 & 0 & 2 & 0 & 2 & 35 \\
\hline & Layered enhancement (target sign) & 0 & 0 & 0 & 4 & 6 & 0 & 0 & 10 \\
\hline & Reduced enhancement & 0 & 0 & 0 & 0 & 0 & 3 & 0 & 3 \\
\hline
\end{tabular}


showed focal bowel wall thickening $(<10 \mathrm{~cm})$ and 9 (17.65\%) showed segmental bowel wall thickening $(>10 \mathrm{~cm})$. Diffuse bowel wall thickening was not seen in any case of adenocarcinoma. Out of 18 cases of infective/ inflammatory bowel lesions, $12(66.67 \%)$ cases showed segmental $(>10 \mathrm{~cm})$ bowel wall thickening, $2(11.11 \%)$ cases of pancolitis showed diffuse bowel wall thickening of colon and $4(22.22 \%)$ cases show focal $(<10 \mathrm{~cm})$ bowel wall thickening.(Table 1$)$

Out of 51 cases of adenocarcinoma of bowel, 31 (60.78\%) showed heterogeneous pattern of post contrast enhancement while 20(39.22\%) showed homogeneous pattern of postcontrast enhancement.Out of 18 cases of infective/ inflammatory bowel lesions, 10 (55.56\%) cases showed layered pattern of enhancement (target sign), 6 (33.33\%) cases showed homogeneous enhancement and 2 (11.11\%) cases showed heterogeneous enhancement. (Table 1)

$12(23.53 \%)$ patients of adenocarcinoma showed exophytic mass, which was not seen in benign conditions. 2 cases of sigmoid diverticulitis were seen and both showed marked asymmetrical bowel wall thickening and inhomogeneous postcontrast enhancement with associated pericolic abscess. Out of 42 patients of colorectal malignancy 18 patients had liver metastasis. 2 patients of amoebic colitis patients had liver abscess.

Out of 47 histopathologically/ follow up imaging proven adenocarcinoma patients, 45 cases were correctly diagnosed with help of multidetector CT scan. Sensitivity for detection of malignant lesions was $95.74 \%$, Specificity was $88.89 \%$ and positive predictive value was $93.75 \%$.Out of 27 histopathologically/ follow up imaging proven cases of benign wall thickening of small bowel and colon, 24 cases were correctly diagnosed with help of multidetector CT scan. Sensitivity was $88.89 \%$, Specificity was $95.74 \%$ and positive predictive value was $92.30 \%$.Out of 74 patients with bowel wall thickening, 69 were correctly diagnosed as benign or malignant with multidetector CT scan. Accuracy was $93.24 \%$.

No single finding was highly specific for diagnosis of bowel malignancy, except asymmetric wall thickening. But combination of multiple characteristic findings was most specific.Findings confirmed by colonoscopy or histopatohology in most of the patients and by peroperative findings, follow up CT scan or sonography in remainder of the patients.Inflammatory conditions showed resolution of disease process by anti-inflammatory medications and antibiotics.

\section{DISCUSSION}

Imaging of small and large bowel is a common procedure in the era of multidetector CT scan. CT colonography (CTC) is a sensitive method to detect colonic polyps and colorectal carcinoma as well as infective and inflammatory conditions of large bowel. The most common age group affected in our study was 40 to 70 years.

Most common bowel pathologies identified in our study were infective and inflammatory lesions (ileocaecal Koch's, infective colitis, inflammatory bowel disease), malignant lesions (adenocarcinoma, lymphoma, carcinoid tumours), colonic polyps, ischemic bowel disease and intussusceptions.
CT Colonography can be performed by two methods. Both techniques are based on distension of the colon for examination of the lumen and wall of the bowel. In the first technique,the bowel lumen is filled with water and intravenous injection of a contrast medium is administered, known as water enema technique. This is used in symptomatic or suspected cases of colorectal carcinoma and for staging of colorectal tumors. ${ }^{1}$ The second technique is the gas enema method of colonography, also called virtual colonoscopy. It is performedby expanding the colon with air or Carbon Dioxide and is done without injection of intravenous contrast medium. It is used as screening tool for detection of polyps. ${ }^{1}$ CT enterography clearly depicts the small bowel inflammation associated with Crohn's disease by displaying mural hyperenhancement, stratification, and thickening; engorged vasa recta; and perienteric inflammatory changes. As a result, CT enterography is becoming the first-line modality for the evaluation of suspected inflammatory bowel disease. $^{2}$

Most of the bowel pathologies manifest as thickening of bowel wall. It is of vital importance to distinguish benign from malignant bowel wall thickening. Certain characteristic of the lesions help differentiate benign and malignant lesions with high degree of accuracy. These characteristics include degree of wall thickening, length of wall thickening, post contrast enhancement pattern, symmetrical or asymmetrical wall thickening and location of the lesion. Using combination of these characteristics, we could diagnose $93.24 \%$ cases correctly as benign or malignant.

Causes of small or large bowel thickening include neoplastic lesions (e.g. adenocarcinoma, lymphoma, carcinoid), infective and inflammatory lesions, ischemic bowel disease. Bowel wall thickening is classified as focal, segmental or diffuse wall thickening. In cases with focal thickening, other associated characteristics like symmetrical or asymmetrical thickening,wall enhancement characteristics and perienteric anomalies allows distinguishing between neoplasms and inflammatory conditions. When there is segmental or diffuse thickening, the pattern of wall enhancement and symmetrical or asymmetrical wall thickening may lead to correct diagnosis. $^{3}$

Acceptable normal thickness of bowel wall depends on degree of distension. Thickness of small bowel wall should not exceed $3 \mathrm{~mm}$ in distended state. Wall thickness of colon may range from 1 to $2 \mathrm{~mm}$ in distended state and up to 5 $\mathrm{mm}$ in collapsed state. $^{3}$ (Figure1) Colonic wall should be barely perceptible if the colon is well distended with contrast material or air. Gas, Feces and minimal fluid are normally present in the colon. ${ }^{4}$

\section{Degree of wall thickening}

Mild thickening of the bowel wall was considered as less than $15 \mathrm{~mm}$ and marked thickening was considered as more than $15 \mathrm{~mm}$. Mild thickening was predominantly seen in infective and inflammatory conditions. Some early malignant lesions also showed mild thickening. Other associated characteristic help in these cases. Focal bowel wall thickening is more commonly seen in adenocarcinoma, while segmental or diffuse thickening is more common in benign infective or inflammatory conditions. ${ }^{5}$ The small bowel 
lymphoma typically shows segmental distribution, which is an exception. ${ }^{6,7}$

Common Inflammatory or infective bowel wall thickening include inflammatory bowel disease (ulcerative colitis and Crohn's disease, infective colitis, amoebic colitis, enteric fever and intestinal tuberculosis. (Figure 2)

In ulcerative colitis, inflammation is limited to mucosa and submucosa, therefore there is mild bowel wall thickening. However, in Crohn's disease there is often transmural inflammation leading to marked bowel wall thickening. One study found the mean thickness of the colon wall in Crohn's disease was $11.0 \mathrm{~mm}$ compared with $7.8 \mathrm{~mm}$ in patients with ulcerative colitis. $^{8}$

Marked bowel wall thickening is commonly seen in malignant etiologies like, adenocarcioma, lymphoma and metastasis. (Figure 3)

\section{Symmetrical or asymmetrical wall thickening}

Symmetrical wall thickening is defined as same degree of wall thickening involving circumference of bowel wall. Asymmetric thickening refers to eccentric thickening along circumference of bowel wall.

Symmetric thickening is seen in intestinal inflammatory conditions, infections, bowel edema, and ischemia. ${ }^{9}$ (figure2, 4) In addition, the bowel is usually symmetrically thickened in cases of submucosal hemorrhage. ${ }^{10,11}$ Some neoplasms may also display symmetric thickening, especially scirrhous carcinoma and occasionally lymphoma ${ }^{9,12}$ (figure5).

Irregular short asymmetric lesions with abrupt margins are the hallmark of adenocarcinomas and metastatic diseases. ${ }^{13}$ (Figure3) In our study $77.77 \%$ of infective/inflammatory lesions showed symmetrical thickening and $84.31 \%$ of adenocarcinoma showed asymmetrical thickening. (Table 1).Asymmetrical wall thickening was more commonly seen in malignant lesions.

\section{Length of involved segment}

Classification of bowel wall thickening as focal $(<10 \mathrm{~cm})$, segmental $(10-30 \mathrm{~cm})$ or diffuse (involving major part of large bowel or small bowel) is crucial. Inflammatory and neoplastic conditions may overlap in length of involvement; evaluation of other characteristics may help to reach the diagnosis. With few exceptions, long segments of involvement are seen in benign conditions. ${ }^{14}$ In our study, Out of 51 cases of adenocarcinoma of bowel, 42 (82.35\%) showed focal bowel wall thickening $(<10 \mathrm{~cm}$ ) (figure 6) and $9(17.65 \%)$ showed segmental bowel wall thickening $(10-30 \mathrm{~cm})$. Diffuse bowel wall thickening was not seen in any case of adenocarcinoma. Out of 18 cases of infective/ inflammatory bowel lesions, $12(66.67 \%)$ cases showed segmental $(10-30 \mathrm{~cm})$ bowel wall thickening, 2 (11.11\%) cases of pancolitis showed diffuse bowel wall thickening of colon and $4(22.22 \%)$ cases show focal $(<10 \mathrm{~cm})$ bowel wall thickening. Focal bowel wall thickening was seen in 2 cases of sigmoid diverticulitis. Common diseases with segmental bowel involvement were infective colitis, ischemic bowel disease. Crohn's disease, ileocaecal Koch's, radiation enteritis and intramural haemorrhage. (Figure7) Diffuse involvement is seen in ulcerative colitis, infectious enteritis, oedema from low-protein states, portal hypertension associated with cirrhosis, low-flow ischaemia and oedema. ${ }^{15}$

\section{Enhancement pattern}

Homogeneous enhancement of bowel wall after intravenous contrast administration is seen in infective/ inflammatory colitis, intestinal tuberculosis, inflammatory bowel diseases (ulcerative colitis and Crohn's disease, submucosal haemorrhage or haematoma and ischemic bowel disease. In case of infarction there may be reduced enhancement if there is necrosis of bowel wall. Inflammatory lesions may show target or double halo sign and stratified enhancement pattern which is a sign of non-malignant disease. ${ }^{16,17,18}$ (Figure 8) The fat halo sign is seen in chronic inflammatory bowel diseases (ulcerative colitis and Crohn's disease). There is fatty infiltration in submucosa with gives the appearance of fat halo sign demonstrating three layers: an inner and outer layer of soft tissue attenuation, between which lies a third layer of fatty attenuation.

In malignant lesions, the markedly thickened bowel wall shows heterogeneous attenuation due to intralesional necrosis. These are most commonly seen in seen in adenocarcinomas and stromal cell tumours. (Figure3) Large size malignant lesions have more tendencies for necrosis and show heterogeneous enhancement. ${ }^{14}$ In our study, there were 2 cases of sigmoid diverticulitis, both showed focal asymmetrical wall thickening with heterogeneous postcontrast enhancement due to adjacent fluid collection and abscess. It becomes frequently difficult to differentiate between malignancies and diverticular disease. However, associated findings help us to differentiate. Pericolonic fat stranding and pericolic abscess are more specific for diverticular disease and usually not seen in malignancy.

In our study, most common histology for malignant lesion was adenocarcinoma and most common site of malignant lesions was colorectal region (82.36\%).Among the colorectal region, rectosigmoid region was most commonly involved (52.38\%).

Acute mesenteric ischemia is a life-threatening condition, with a reported mortality rate of 50-90\%. Therefore, it requires early diagnosis and treatment. MDCT technology has dramatically improved the performance of CT by allowing rapid volumetric data acquisition to provide increased longitudinal spatial resolution over a large anatomic volume. From the volume data, multiplanar reformatted images and CT angiograms with $2 \mathrm{D}$ or $3 \mathrm{D}$ visualization can be obtained. The rapid scanning capability of this technique coupled with IV bolus contrast injection substantially optimizes scan timing to allow both the arterial and venous phases to be imaged. These advantages of recent CT technology are extremely helpful in diagnosis of mesentery ischemia along with identification of mesenteric vascular thrombus, other causes of bowel ischemia, abnormalities of bowel wall and mesentery. With recent generation of multidetector CT scanners, the ability of CT for diagnosing mesenteric ischemia has recently been reported to have a sensitivity of approximately 90\%. Also apart from diagnosis of mesenteric ischemia, CT is extremely useful to provide alternative cause of patient's symptoms in the acute setting when mesenteric ischemia is suspected. ${ }^{19}$

Most common locations include splenic flexure and 
rectosigmoid. Segmental wall thickening with the layered appearance as target sign is seen on enhanced scan. Pneumatosis may be seen in advance disease. Complications include infarction, perforation and stricture. In first stage immediately after occlusion thrombus can be seen in superior mesenteric artery (SMA) or superior mesenteric vein $(\mathrm{SMV})$ but wall changes are not developed. In second stage, mild distension of bowel wall with paperthin wall and mural enhancement is seen. In case of SMV thrombosis mural thickening, mural hemorrhage and sub mucosal edema results in target appearance of the bowel wall. The damage at this stage is reversible and three outcomes can ensue: healing which may lead to stricture formation, chronic ischemia or progress to infarction. ${ }^{15}$

In our study, Out of 5 patients with ischemic colitis, CT angiography detected superior mesenteric artery thrombosis in four patients and portal vein thrombosis and superior mesenteric vein thrombosis in one patient. (Figure9) Out of 5 cases of ischemic bowel lesions, mild wall thickening (80\%), symmetrical thickening (100\%), reduced enhancement (60\%) and segmental involvement (80\%) were seen.

Imaging findings of acute appendicitis include are dilated appendix greater than $0.6 \mathrm{~cm}$ diameter, circumferential wall thickening, and wall enhancement after contrast material administration and periappendicial fat stranding. Radioopqaue appendicolith is occasionally seen making the diagnosis more certain. Other conditions like intussusceptions and volvulus are easy to diagnose. In adults, mass can be the lead point of intussusceptions. ${ }^{20}$

\section{CONCLUSION}

Thickening of small and large bowel wall is seen in variety of conditions. No single characteristic is highly specific for differentiation between benign and malignant lesions. Careful analysis of characteristic of lesions on CT scan including degree of bowel wall thickening, symmetrical or asymmetrical thickening, length of bowel wall thickening, pattern of bowel wall enhancement and location of the lesion help to narrow down the differential diagnosis. Thus, multidetector CT scan is "The imaging modality of choice" for characterization of bowel lesions.

\section{REFERENCES}

1. Ridereau-Zins C, Pilleul F, Gandon Y, Laurent V. CT colonography: Why? when? how? Diagnostic and interventional imaging. 2012; 93(1): 2-9.

2. Paulsen SR, Huprich JE, Fletcher JG, et al. CT Enterography as a Diagnostic Tool in Evaluating Small Bowel Disorders: Review of Clinical Experience with over 700 Cases1. RadioGraphics. 2006; 26(3):641-57.

3. Fernandes T, Oliveira MI, Castro R, Araújo B, Viamonte B, Cunha R. Bowel wall thickening at CT: Simplifying the diagnosis. Insights Imaging. 2014; 5(2):195-208.

4. Horton KM, Corl FM, Fishman EK. CT Evaluation of the Colon: Inflammatory Disease. RadioGraphics. 2000; 20 (2):399-418.

5. Chou CK, Reng HW, Mak CW, Lin MP. Clinical significance of poor CT enhancement of the thickened small-bowel wall in patients with acute abdominal pain. American Journal of Roentgenology. 2006; 186 (2):491-
8.

6. Macari M, Megibow AJ, Balthazar EJ. A pattern approach to the abnormal small bowel: Observations at MDCT and CT enterography. American Journal of Roentgenology. 2007;188 (5):1344-55.

7. Wittenberg J, Harisinghani MG, Jhaveri K, Varghese J, Mueller PR. Algorithmic approach to CT diagnosis of the abnormal bowel wall. Radiographics. 2002; 22 (5):1093-107.

8. Philpotts, L. E., Heiken, J. P., Westcott, M. A., \& Gore, R. M. (1994). Colitis: use of CT findings in differential diagnosis. Radiology. 1994;190(2):445-9.

9. Balthazar, E. J. (1991). CT of the gastrointestinal tract: Principles and interpretation. American Journal of Roentgenology. 2006;186(2): 491-498.

10. Lane, M. J., Katz, D. S., Mindelzun, R. E., \& Jeffrey, R. B. Spontaneous intramural small bowel haemorrhage: importance of non-contrast CT. Clinical Radiology. May 1997; 52 (5): 378-380.

11. Balthazar, E. J., Hulnick, D., Megibow, A. J., \& Opulencia, J. F. Computed tomography of intramural intestinal hemorrhage and bowel ischemia. Journal of Computer Assisted Tomography. Jan 1987; 11(1):67-72.

12. Balthazar, E. J., Noordhoorn, M., Megibow, A. J., \& Gordon, R. B. CT of small-bowel lymphoma in immunocompetent patients and patients with AIDS: Comparison of findings. American Journal of Roentgenology. 1997;168 (3):675-80.

13. Buckley, J. A., \& Fishman, E. K. CT evaluation of small bowel neoplasms: spectrum of disease. Radiographics. 1998; 18 (2):379-92.

14. Macari, M., \& Balthazar, E. J. CT of bowel wall thickening: Significance and pitfalls of interpretation. American Journal of Roentgenology. 2001;176 (5):1105-16.

15. Bhatt, C. J., Patel, L. N., Baraiya, M., Patel, K. K., Vaishnav, K. U., \& Shah, D. S. Multidetector Computed Tomography in Large Bowel Lesions-A Study of 100 Cases. The Indian Journal of Surgery. 2011; 73(5), 352-358.

16. Frager DH, Goldman M, Beneventano TC. Computed tomography in Crohn disease. Journal of Computer Assisted Tomography. 1983; 7(5):819-824.

17. Jones B, Fishman EK, Hamilton SR, et al. Submucosal accumulation of fat in inflammatory bowel disease: CT/ pathologic correlation. Journal of Computer Assisted Tomography. 1986; 10 (5):759-763.

18. Ahualli, J. The target sign: bowel wall. Radiology. 2005; 234(2): 549-550.

19. Furukawa, A., Kanasaki, S., Kono, N., Wakamiya, M., Tanaka, T., Takahashi, M., \& Murata, K. CT Diagnosis of Acute Mesenteric Ischemia from Various Causes. American Journal of Roentgenology. 2009; 192(2): 408-416.

20. Curtin, K. R., Fitzgerald, S. W., Nemcek Jr, A. A., Hoff, F. L., \& Vogelzang, R. L. CT diagnosis of acute appendicitis: imaging findings. American journal of roentgenology. 1995;164 (4):905-909.

\section{Source of Support: Nil; Conflict of Interest: None}

Submitted: 06-08-2018; Accepted: 30-08-2018; Published online: 19-09-2018 